Kragujevac Journal of Mathematics

Volume 44(3) (2020), Pages 401-413.

\title{
BEST PROXIMITY POINT RESULTS VIA SIMULATION FUNCTIONS IN METRIC-LIKE SPACES
}

\author{
G. V. V. J. RAO ${ }^{1}$, H. K. NASHINE ${ }^{2 *}$, AND Z. KADELBURG ${ }^{3}$ \\ ABSTRACT. In this paper, we discuss the existence of best proximity points of certain \\ mappings via simulation functions in the frame of complete metric-like spaces. Some \\ consequences and examples are given of the obtained results.
}

\section{INTRODUCTION}

Khojasteh et al. introduced in [13] the notion of simulation function in order to unify several fixed point results obtained by various authors. These functions were later utilized by Karapinar and Khojasteh in [9] to solve some problems concerning best proximity points.

On the other hand, spaces more general than metric and fixed point and related problems in them have been lately a wide field of interest of huge number of mathematicians. Among them, metric-like spaces, introduced by Amini-Harandi in [2], took a prominent place.

In this paper, we are going to extend these investigations to best proximity points of mappings acting in complete metric-like spaces, using conditions involving simulation functions. The results will be illustrated by several examples, showing the strength of these results compared with others existing in the literature.

\section{Preliminaries}

Throughout the paper, $\mathbb{R}$ and $\mathbb{R}^{+}, \mathbb{R}_{0}^{+}$will denote the set of real numbers, the set of positive real numbers and the set of nonnegative real numbers, respectively. Also, $\mathbb{N}_{0}$ and $\mathbb{N}$ will denote the set of nonnegative, resp. positive integers.

Key words and phrases. Z-contraction, best proximity point, simulation function, admissible mapping.

2010 Mathematics Subject Classification. Primary: 41A50. Secondary: 47H10, 54H25.

DOI 10.46793/KgJMat2003.401R

Received: April 14, 2018.

Accepted: May 22, 2018. 
We shall first recall some basic definitions and some results from $[1,5,13]$.

Definition 2.1 ([13]). A simulation function is a mapping $\zeta: \mathbb{R}_{0}^{+} \times \mathbb{R}_{0}^{+} \rightarrow \mathbb{R}$ satisfying the following conditions:

$\left(\zeta_{1}\right) \zeta(0,0)=0$

$\left(\zeta_{2}\right) \zeta(t, s)<s-t$ for all $t, s>0$

$\left(\zeta_{3}\right)$ if $\left\{t_{n}\right\}$ and $\left\{s_{n}\right\}$ are sequences in $(0, \infty)$ such that $\lim _{n \rightarrow \infty} t_{n}=\lim _{n \rightarrow \infty} s_{n}=$ $l \in(0, \infty)$, then $\lim \sup _{n \rightarrow \infty} \zeta\left(t_{n}, s_{n}\right)<0$.

Note that, according to the axiom $\left(\zeta_{2}\right)$, each simulation function $\zeta$ satisfies $\zeta(t, t)<0$ for all $t>0$. The family of all simulation functions will be denoted by $z$.

Example 2.1 (See, e.g., $[1,5,7,13]$ ). For $i=1,2, \ldots, 6$, define mappings $\zeta_{i}: \mathbb{R}_{0}^{+} \times \mathbb{R}_{0}^{+} \rightarrow$ $\mathbb{R}$, as follows.

(i) $\zeta_{1}(t, s)=\phi_{1}(s)-\phi_{2}(t)$ for all $t, s \in \mathbb{R}_{0}^{+}$, where $\phi_{1}, \phi_{2}: \mathbb{R}_{0}^{+} \rightarrow \mathbb{R}_{0}^{+}$are continuous functions, with $\phi_{i}(t)=0$ if and only if $t=0$ and $\phi_{1}(t)<t \leq \phi_{2}(t)$ for all $t>0$.

(ii) $\zeta_{2}(t, s)=s-\frac{f(t, s)}{g(t, s)} t$ for all $t, s \in \mathbb{R}_{0}^{+}$, where $f, g: \mathbb{R}_{0}^{+2} \rightarrow \mathbb{R}_{0}^{+}$are two functions, continuous with respect to each variable and such that $f(t, s)>g(t, s)$ for all $t, s>0$.

(iii) $\zeta_{3}(t, s)=s-\phi(s)-t$ for all $t, s \in \mathbb{R}_{0}^{+}$, where $\phi: \mathbb{R}_{0}^{+} \rightarrow \mathbb{R}_{0}^{+}$is a continuous functions, with $\phi(t)=0$ if and only if $t=0$.

(iv) If $\varphi: \mathbb{R}_{0}^{+} \rightarrow[0,1)$ is a function such that $\lim _{\sup _{t \rightarrow r^{+}}} \varphi(t)<1$ for all $r>0$, let

$$
\zeta_{4}(t, s)=s \varphi(s)-t, \quad \text { for all } t, s \in \mathbb{R}_{0}^{+} .
$$

(v) If $\eta: \mathbb{R}_{0}^{+} \rightarrow \mathbb{R}_{0}^{+}$is an upper semi-continuous function such that $\eta(t)<t$ for all $t>0$ and $\eta(0)=0$, let

$$
\zeta_{5}(t, s)=\eta(s)-t, \quad \text { for all } t, s \in \mathbb{R}_{0}^{+} .
$$

(vi) If $\phi: \mathbb{R}_{0}^{+} \rightarrow \mathbb{R}_{0}^{+}$is a function such that $\int_{0}^{\epsilon} \phi(u) d u>\epsilon$ for each $\epsilon>0$, let

$$
\zeta_{6}(t, s)=s-\int_{0}^{t} \phi(u) d u, \quad \text { for all } t, s \in \mathbb{R}_{0}^{+} .
$$

It is clear that each function $\zeta_{i}, i=1,2, \ldots, 6$, is a simulation function.

Definition $2.2([2])$. Let $X$ be a nonempty set, and a mapping $\sigma: X \times X \rightarrow \mathbb{R}_{0}^{+}$is such that, for all $x, y, z \in X$,

$\left(\sigma_{1}\right) \sigma(x, y)=0$ implies $x=y$;

$\left(\sigma_{2}\right) \sigma(x, y)=\sigma(y, x)$;

$\left(\sigma_{3}\right) \sigma(x, y) \leq \sigma(x, z)+\sigma(z, y)$.

Then $(X, \sigma)$ is said to be a metric-like space.

As is well known, each partial metric space is an example of a metric-like space. The converse is not true. The following example illustrates this statement. 
Example 2.2. Take $X=\{1,2,3\}$ and consider the metric-like $\sigma: X \times X \rightarrow \mathbb{R}_{0}^{+}$given by

$$
\begin{gathered}
\sigma(1,1)=0, \quad \sigma(2,2)=1, \quad \sigma(3,3)=\frac{2}{3}, \\
\sigma(2,1)=\sigma(1,2)=\frac{9}{10}, \quad \sigma(1,3)=\sigma(3,1)=\frac{7}{10}, \quad \sigma(2,3)=\sigma(3,2)=\frac{4}{5} .
\end{gathered}
$$

Since $\sigma(2,2) \neq 0, \sigma$ is not a metric and since $\sigma(2,2)>\sigma(2,1), \sigma$ is not a partial metric.

Every metric-like $\sigma$ on $X$ generates a topology $\tau_{\sigma}$ whose base is the family of all open $\sigma$-balls

$$
\left\{B_{\sigma}(x, \delta): x \in X, \delta>0\right\}
$$

where $B_{\sigma}(x, \delta)=\{y \in X:|\sigma(x, y)-\sigma(x, x)|<\delta\}$, for all $x \in X$ and $\delta>0$.

Definition $2.3([2])$. Let $(X, \sigma)$ be a metric-like space, let $\left\{x_{n}\right\}$ be a sequence in $X$ and $x \in X$. Then

(i) $\left\{x_{n}\right\}$ is said to converge to $x$, w.r.t. $\tau_{\sigma}$, if $\lim _{n \rightarrow \infty} \sigma\left(x_{n}, x\right)=\sigma(x, x)$;

(ii) $\left\{x_{n}\right\}$ is called a Cauchy sequence in $(X, \sigma)$ if $\lim _{n, m \rightarrow \infty} \sigma\left(x_{n}, x_{m}\right)$ exists (and is finite);

(iii) $(X, \sigma)$ is called complete if every Cauchy sequence $\left\{x_{n}\right\}$ in $X$ converges with respect to $\tau_{\sigma}$ to a point $x \in X$ such that

$$
\lim _{n, m \rightarrow \infty} \sigma\left(x_{n}, x_{m}\right)=\lim _{n \rightarrow \infty} \sigma\left(x_{n}, x\right)=\sigma(x, x) ;
$$

(iv) a function $f: X \rightarrow X$ is continuous if for any sequence $\left\{x_{n}\right\}$ in $X$ such that $\sigma\left(x_{n}, x\right) \rightarrow \sigma(x, x)$ as $n \rightarrow \infty$, we have $\sigma\left(f x_{n}, f x\right) \rightarrow \sigma(f x, f x)$ as $n \rightarrow \infty$.

Note that the limit of a sequence in a metric-like space might not be unique.

Lemma 2.1 ([11]). Let $(X, \sigma)$ be a metric-like space. Let $\left\{x_{n}\right\}$ be a sequence in $X$ such that $x_{n} \rightarrow x$ where $x \in X$ and $\sigma(x, x)=0$. Then for all $y \in X$, we have

$$
\lim _{n \rightarrow \infty} \sigma\left(x_{n}, y\right)=\sigma(x, y) \text {. }
$$

$\Psi$ will denote the family of non-decreasing functions $\psi: \mathbb{R}_{0}^{+} \rightarrow \mathbb{R}_{0}^{+}$satisfying the following conditions:

(i) $\psi(t)<t$, for any $t \in \mathbb{R}^{+}$;

(ii) $\psi$ is continuous at 0 .

Let $(X, \sigma)$ be a metric-like space, and $U$ and $V$ be two non-empty subsets of $X$. Recall the following standard notation:

$$
\begin{aligned}
\sigma(U, V) & :=\inf \{\sigma(u, v): u \in U, v \in V\}, \\
U_{0} & :=\{u \in U: \sigma(u, v)=\sigma(U, V) \text { for some } v \in V\}, \\
V_{0} & :=\{v \in V: \sigma(u, v)=\sigma(U, V) \text { for some } u \in U\} .
\end{aligned}
$$


Consider now a non-self mapping $T: U \rightarrow V$ and the equation $T u=u(u \in U)$. As is well known, a solution of this equation, if it exists, is called a fixed point of $T$. If such solution does not exist, an approximate solution $u^{*} \in U$ have the least possible error when $\sigma\left(u^{*}, T u^{*}\right)=\sigma(U, V)$. In this case, $u^{*}$ is called a best proximity point of the mapping $T: U \rightarrow V$.

Finally, recall the following useful notions.

Definition $2.4([6])$. Let $U$ and $V$ be nonempty subsets of a metric-like space $(X, \sigma)$, and $\alpha: U \times U \rightarrow \mathbb{R}_{0}^{+}$be a function. We say that the mapping $T$ is $\alpha$-proximal admissible if

$$
\alpha(x, y) \geq 1 \text { and } \sigma(u, T x)=\sigma(v, T y)=\sigma(U, V) \Rightarrow \alpha(u, v) \geq 1,
$$

for all $x, y, u, v \in X$.

If $\sigma(U, V)=0$, then $T$ reduces from $\alpha$-proximal admissible to $\alpha$-admissible.

Definition $2.5([8,10])$. Let $T: X \rightarrow X$ be a mapping and $\alpha: X \times X \rightarrow \mathbb{R}_{0}^{+}$be a function. We say that the mapping $T$ is triangular weakly- $\alpha$-admissible if

$$
\alpha(x, y) \geq 1 \text { and } \alpha(y, z) \geq 1 \Rightarrow \alpha(x, z) \geq 1 \text {. }
$$

\section{Main Results}

Definition 3.1. Let $(X, \sigma)$ be a metric-like space, $U$ and $V$ be two non-empty subsets of $X, \psi \in \Psi, \alpha: X \times X \rightarrow \mathbb{R}_{0}^{+}$and $\zeta \in z$. We say that $T: U \rightarrow V$ is an $\alpha-\psi-\zeta-$ contraction if $T$ is $\alpha$-proximal admissible and

$\alpha(x, y) \geq 1$ and $\sigma(u, T x)=\sigma(v, T y)=\sigma(U, V) \Rightarrow \zeta(\alpha(x, y) \sigma(u, v), \psi(\sigma(x, y))) \geq 0$,

for all $x, y, u, v \in U$.

Definition 3.2. Let $(X, \sigma)$ be a metric-like space, $U$ and $V$ be two non-empty subsets of $X, \alpha: X \times X \rightarrow \mathbb{R}_{0}^{+}$and $\zeta \in z$. We say that $T: U \rightarrow V$ is an $\alpha$ - $\zeta$-contraction if $T$ is $\alpha$-proximal admissible and

$$
\alpha(x, y) \geq 1 \text { and } \sigma(u, T x)=\sigma(v, T y)=\sigma(U, V) \Rightarrow \zeta(\alpha(x, y) \sigma(u, v), \sigma(x, y)) \geq 0,
$$

for all $x, y, u, v \in U$.

Notice that Definition 3.2 is not a special case of Definition 3.1 since the function $\psi(t)=t$ does not belong to $\Psi$.

The following lemma provides a standard step in proving that the given sequence is Cauchy in a certain space.

Lemma 3.1 (See, e.g., [14]). Let $(X, \sigma)$ be a metric-like space and let $\left\{x_{n}\right\}$ be a sequence in $X$ such that $\sigma\left(x_{n+1}, x_{n}\right)$ is non-increasing and that $\lim _{n \rightarrow \infty} \sigma\left(x_{n+1}, x_{n}\right)=0$. If $\left\{x_{n}\right\}$ is not a Cauchy sequence, then there exist an $\epsilon>0$ and two sequences $\left\{m_{k}\right\}$ 
and $\left\{n_{k}\right\}$ of positive integers such that the following four sequences tend to $\epsilon$ when $k \rightarrow \infty$ :

$$
\sigma\left(x_{m_{k}}, x_{n_{k}}\right), \sigma\left(x_{m_{k}+1}, x_{n_{k}+1}\right), \sigma\left(x_{m_{k}-1}, x_{n_{k}}\right), \sigma\left(x_{m_{k}}, x_{n_{k}-1}\right) .
$$

Now we present the main results of this article.

Theorem 3.1. Let $(X, \sigma)$ be a metric-like space, $U$ and $V$ be two non-empty subsets of $X, \alpha: X \times X \rightarrow \mathbb{R}_{0}^{+}, \psi \in \Psi$ and $\zeta \in \mathcal{Z}$ is non-decreasing with respect to its second argument. Suppose that $T: U \rightarrow V$ is an $\alpha-\psi-\zeta$-contraction and

(1) $T$ is triangular weakly- $\alpha$-admissible;

(2) $U$ is closed with respect to the topology $\tau_{\sigma}$;

(3) $T\left(U_{0}\right) \subset V_{0}$;

(4) there exist $x_{0}, x_{1} \in U$ such that $\sigma\left(x_{1}, T x_{0}\right)=\sigma(U, V)$ and $\alpha\left(x_{0}, x_{1}\right) \geq 1$;

(5) $T$ is continuous.

Then, $T$ has a best proximity point, that is, there exists $z \in U$ such that $\sigma(z, T z)=$ $\sigma(U, V)$.

Proof. Take $x_{0}, x_{1} \in U$ given as in (4). Taking (3) into account, we conclude that $T x_{1} \in V_{0}$ which implies that there exists $x_{2} \in U$ such that $\sigma\left(x_{2}, T x_{1}\right)=\sigma(U, V)$. Since $\alpha\left(x_{0}, x_{1}\right) \geq 1$ and $T$ is $\alpha$-proximal admissible, we conclude that $\alpha\left(x_{1}, x_{2}\right) \geq 1$. Recursively, a sequence $\left\{x_{n}\right\} \subset U$ can be chosen satisfying

$$
\sigma\left(x_{n+1}, T x_{n}\right)=\sigma(U, V) \text { and } \alpha\left(x_{n}, x_{n+1}\right) \geq 1, \quad \text { for all } n \in \mathbb{N}_{0} .
$$

If $x_{k}=x_{k+1}$ for some $k \in \mathbb{N}_{0}$, then $\sigma\left(x_{k}, T x_{k}\right)=\sigma\left(x_{k+1}, T x_{k}\right)=\sigma(U, V)$, meaning that $x_{k}$ is the required best proximal point. Hence, we will further assume that

$$
x_{n} \neq x_{n+1}, \quad \text { for all } n \in \mathbb{N}_{0} .
$$

Using relations (3.3) and (3.4), we get that $\sigma\left(x_{n}, T x_{n-1}\right)=\sigma\left(x_{n+1}, T x_{n}\right)=\sigma(U, V)$, for all $n \in \mathbb{N}$. Furthermore, by (3.1)

$$
\zeta\left(\alpha\left(x_{n-1}, x_{n}\right) \sigma\left(x_{n}, x_{n+1}\right), \psi\left(\sigma\left(x_{n-1}, x_{n}\right)\right)\right) \geq 0, \quad \text { for all } n \in \mathbb{N},
$$

since $T: U \rightarrow V$ is an $\alpha-\psi-\zeta$-contraction. Regarding (3.4) and $\left(\zeta_{2}\right)$, the inequality (3.5) implies that

$$
\sigma\left(x_{n}, x_{n+1}\right) \leq \alpha(x, y) \sigma\left(x_{n}, x_{n+1}\right) \leq \psi\left(\sigma\left(x_{n-1}, x_{n}\right)\right)<\sigma\left(x_{n-1}, x_{n}\right), \quad \text { for all } n \in \mathbb{N} .
$$

Thus, $\left\{\sigma\left(x_{n}, x_{n+1}\right)\right\}$ is a non-increasing sequence bounded from below and there exists $L \in \mathbb{R}_{0}^{+}$such that $\sigma\left(x_{n}, x_{n+1}\right) \rightarrow L$ as $n \rightarrow \infty$. We shall prove that $L=0$. Suppose, on the contrary, that $L>0$. Taking the upper limit in (3.5) as $n \rightarrow \infty$, regarding $\left(\zeta_{3}\right)$, property (i) of $\psi \in \Psi$ and that $\zeta$ is non-decreasing with respect to the second argument, we deduce

$$
\begin{aligned}
0 & \leq \limsup _{n \rightarrow \infty} \zeta\left(\alpha\left(x_{n}, x_{n-1}\right) \sigma\left(x_{n}, x_{n+1}\right), \psi\left(\sigma\left(x_{n}, x_{n-1}\right)\right)\right) \\
& \leq \limsup _{n \rightarrow \infty} \zeta\left(\alpha\left(x_{n}, x_{n-1}\right) \sigma\left(x_{n}, x_{n+1}\right), \sigma\left(x_{n}, x_{n-1}\right)\right)<0,
\end{aligned}
$$

which is a contradiction. We conclude that $\lim _{n \rightarrow \infty} \sigma\left(x_{n}, x_{n+1}\right)=0$. 
We shall now prove that the sequence $\left\{x_{n}\right\}$ is Cauchy. Suppose that it is not. Then, there exist $\epsilon>0$ and subsequences $\left\{x_{m_{k}}\right\}$ and $\left\{x_{m_{k}}\right\}$ of $\left\{x_{n}\right\}$, so that $n_{k}>m_{k}>k$ and

$$
\sigma\left(x_{m_{k}}, x_{n_{k}}\right) \geq \epsilon \text { and } \sigma\left(x_{m_{k}}, x_{n_{k}-1}\right)<\epsilon .
$$

By Lemma 2.1, we have

$$
\lim _{k \rightarrow \infty} \sigma\left(x_{m_{k}}, x_{n_{k}}\right)=\lim _{k \rightarrow \infty} \sigma\left(x_{n_{k}-1}, x_{m_{k}-1}\right)=\epsilon .
$$

Since $T$ is triangular weakly- $\alpha$-admissible, from (3.3), we get that

$$
\alpha\left(x_{n}, x_{m}\right) \geq 1, \quad \text { for all } n, m \in \mathbb{N}_{0} \text { with } n>m .
$$

Hence,

$$
\alpha\left(x_{m_{k}}, x_{n_{k}}\right) \geq 1 \text { and } \sigma\left(x_{m_{k}}, T x_{m_{k}-1}\right)=\sigma\left(x_{n_{k}}, T x_{n_{k}-1}\right)=\sigma(U, V), \quad \text { for all } k \in \mathbb{N} .
$$

Since $T$ is an $\alpha-\psi-\zeta$-contraction, the obtained relations (3.7) yield the following inequality:

$$
0 \leq \zeta\left(\alpha\left(x_{n}, x_{n-1}\right) \sigma\left(x_{m_{k}}, x_{n_{k}}\right), \psi\left(\sigma\left(x_{m_{k}}, x_{n_{k}}\right)\right)\right), \quad \text { for all } k \in \mathbb{N} .
$$

Letting $k \rightarrow \infty$, using (3.6) and $\left(\zeta_{3}\right)$, and regarding properties of $\psi \in \Psi$ and that $\zeta$ is non-decreasing with respect to the second argument, we obtain

$$
\begin{aligned}
0 & \leq \limsup _{n \rightarrow \infty} \zeta\left(\alpha\left(x_{n}, x_{n-1}\right) \sigma\left(x_{m_{k}}, x_{n_{k}}\right), \psi\left(\sigma\left(x_{m_{k}-1}, T x_{n_{k}-1}\right)\right)\right) \\
& \leq \limsup _{n \rightarrow \infty} \zeta\left(\alpha\left(x_{n}, x_{n-1}\right) \sigma\left(x_{m_{k}}, x_{n_{k}}\right), \sigma\left(x_{m_{k}-1}, T x_{n_{k}-1}\right)\right)<0,
\end{aligned}
$$

which is a contradiction. Thus, we conclude that the sequence $\left\{x_{n}\right\}$ is Cauchy in $U$.

Since $U$ is a closed subset of a complete metric-like space $(X, \sigma)$, there exists $z \in U$ such that

$$
\lim _{n \rightarrow \infty} \sigma\left(x_{n}, z\right)=0 .
$$

Since $T$ is continuous, we deduce that

$$
\lim _{n \rightarrow \infty} \sigma\left(T x_{n}, T z\right)=0 .
$$

From (3.3), using the triangle inequality together with (3.8) and (3.9), we find that

$$
\sigma(U, V)=\lim _{n \rightarrow \infty} \sigma\left(x_{n+1}, T x_{n}\right)=\sigma(z, T z) .
$$

Thus, $z \in U$ is a best proximity point of the mapping $T$.

The continuity hypothesis in Theorem 3.1 can be omitted if we assume the following additional condition on $U$ :

$(P)$ if a sequence $\left\{u_{n}\right\}$ in $U$ converges to $u \in U$ and is such that $\alpha\left(u_{n}, u_{n+1}\right) \geq 1$ for $n \geq 1$, then there is a subsequence $\left\{u_{n(k)}\right\}$ of $\left\{u_{n}\right\}$ with $\alpha\left(u_{n(k)}, u\right) \geq 1$ for all $k$. 
Theorem 3.2. Let all the conditions of Theorem 3.1 hold, except that the condition (5) is replaced by

(5') (P) holds.

Then $T$ has a best proximity point.

Proof. As in the proof of Theorem 3.1 we conclude that there exists a sequence $\left\{x_{n}\right\}$ in $U_{0}$ which converges to $z \in U_{0}$. Using (3), we note that $T z \in V_{0}$ and hence

$$
\sigma\left(u_{1}, T z\right)=\sigma(U, V), \quad \text { for some } u_{1} \in U_{0} .
$$

Notice that from $(P)$, we have $\alpha\left(x_{n_{k}}, z\right) \geq 1$ for all $k \in \mathbb{N}$. Since $T$ is $\alpha$-proximal admissible and

$$
\sigma\left(u_{1}, T z\right)=\sigma\left(x_{n_{k}+1}, T x_{n_{k}}\right)=\sigma(U, V),
$$

we obtain that $\alpha\left(x_{n_{k}+1}, u_{1}\right) \geq 1$ for all $k \in \mathbb{N}$ and

$$
\zeta\left(\alpha\left(x_{n_{k}+1}, u_{1}\right) \sigma\left(u_{1}, x_{n_{k}+1}\right), \psi\left(\sigma\left(z, x_{n_{k}}\right)\right)\right) \geq 0 .
$$

Then, $\left(\zeta_{2}\right)$ implies that

$$
\sigma\left(u_{1}, x_{n_{k}+1}\right) \leq \alpha\left(x_{n_{k}+1}, u_{1}\right) \sigma\left(u_{1}, x_{n_{k}+1}\right) \leq \psi\left(\sigma\left(z, x_{n_{k}}\right)\right)<\sigma\left(z, x_{n_{k}}\right)
$$

and so $\lim _{k \rightarrow \infty} \sigma\left(u_{1}, x_{n_{k}+1}\right) \rightarrow 0$. Thus, $u_{1}=z$ and by (3.10) we have $\sigma(z, T z)=$ $\sigma(U, V)$.

Theorem 3.3. Let $(X, \sigma)$ be a metric-like space, $U$ and $V$ be two non-empty subsets of $X, \zeta \in \mathcal{Z}$ and $\alpha: X \times X \rightarrow \mathbb{R}_{0}^{+}$. Suppose that $T: U \rightarrow V$ is an $\alpha$ - $\zeta$-contraction and that conditions (1)-(4) of Theorem 3.1 are satisfied, as well as

$\left(5^{\prime \prime}\right) T$ is continuous or $(\mathrm{P})$ holds.

Then, $T$ has a best proximity point.

Proof. By following the lines in the proof of Theorem 3.1, we easily construct a sequence $\left\{x_{n}\right\}$ in $U$ which converges to some $z \in U$, moreover

$$
\lim _{n \rightarrow \infty} \sigma\left(x_{n}, z\right)=0 .
$$

Suppose first that $T$ is continuous. Then

$$
\lim _{n \rightarrow \infty} \sigma\left(T x_{n}, T z\right)=0 .
$$

From (3.3), the triangle inequality together with (3.11) and (3.12) imply

$$
\sigma(U, V)=\lim _{n \rightarrow \infty} \sigma\left(x_{n+1}, T x_{n}\right)=\sigma(z, T z) .
$$

In other words, $z \in U$ is a best proximity of the mapping $T$.

Suppose now that (P) holds. Regarding (3), we note that $T z \in V_{0}$ and hence

$$
\sigma\left(u_{1}, T z\right)=\sigma(U, V), \quad \text { for some } u_{1} \in U_{0} .
$$

Notice that from $(P)$, we have $\alpha\left(x_{n_{k}}, z\right) \geq 1$ for all $k \in \mathbb{N}$. Since $T$ is $\alpha$-proximal admissible, and

$$
\sigma\left(u_{1}, T z\right)=\sigma\left(x_{n_{k}+1}, T x_{n_{k}}\right)=\sigma(U, V)
$$


we get that $\alpha\left(x_{n_{k}+1}, u_{1}\right) \geq 1$ for all $k \in \mathbb{N}$ and

$$
\zeta\left(\alpha\left(x_{n_{k}+1}, u_{1}\right) \sigma\left(u_{1}, x_{n_{k}+1}\right), \sigma\left(z, x_{n_{k}}\right)\right) \geq 0 .
$$

Then, $\left(\zeta_{2}\right)$ implies that $\sigma\left(u_{1}, x_{n_{k}+1}\right) \leq \alpha\left(x_{n_{k}+1}, u_{1}\right) \sigma\left(u_{1}, x_{n_{k}+1}\right) \leq \sigma\left(z, x_{n_{k}}\right)$ and so

$$
\lim _{k \rightarrow \infty} \sigma\left(u_{1}, x_{n_{k}+1}\right) \rightarrow 0 .
$$

Thus, $u_{1}=z$ and by (3.13) we have $\sigma(z, T z)=\sigma(U, V)$ and the proof is completed.

Notice that Theorem 3.3 cannot be obtained by combining Theorems 3.1 and 3.2, since the function $\psi(t)=t$ does not belong to $\Psi$. Furthermore, in Theorems 3.1 and 3.2 , we have an additional condition that $\zeta$ is non-decreasing in its second argument.

Definition 3.3. Let $(X, \sigma)$ be a metric-like space, $U$ and $V$ be two non-empty subsets of $X, \alpha: X \times X \rightarrow \mathbb{R}_{0}^{+}$and $\zeta \in z$. We say that $T: U \rightarrow V$ is a generalized $\alpha-\zeta$ contraction if $T$ is $\alpha$-proximal admissible and

$$
\alpha(x, y) \geq 1 \text { and } \sigma(u, T x)=\sigma(v, T y)=\sigma(U, V) \Rightarrow \zeta(\alpha(x, y) \sigma(u, v), r(x, y)) \geq 0,
$$

for all $x, y, u, v \in U$ with $x \neq y$, where

$$
r(x, y)=\max \left\{\sigma(x, y), \frac{\sigma(x, u) \sigma(y, v)}{\sigma(x, y)}\right\} .
$$

Theorem 3.4. Let $(X, \sigma)$ be a metric-like space, $U$ and $V$ be two non-empty subsets of $X$ and $\alpha: X \times X \rightarrow \mathbb{R}_{0}^{+}, \zeta \in Z$. Suppose that $T: U \rightarrow V$ is a generalized $\alpha-\zeta$-contraction and conditions (1)-(5) of Theorem 3.1 are satisfied. Then $T$ has a best proximity point.

Proof. As in the proof of Theorem 3.1, we can construct a sequence $\left\{x_{n}\right\}$ in $X$ satisfying conditions (3.3) and (3.4). Combining these relations with (3.14), we get that $\sigma\left(x_{n}, T x_{n-1}\right)=\sigma\left(x_{n+1}, T x_{n}\right)=\sigma(U, V)$ for all $n \in \mathbb{N}$ and

$$
\zeta\left(\alpha\left(x_{n-1}, x_{n}\right) \sigma\left(x_{n}, x_{n+1}\right), r\left(x_{n-1}, x_{n}\right)\right) \geq 0, \quad \text { for all } n \in \mathbb{N} .
$$

Here,

$$
\begin{aligned}
r\left(x_{n-1}, x_{n}\right) & =\max \left\{\frac{\sigma\left(x_{n-1}, x_{n}\right) \sigma\left(x_{n}, x_{n+1}\right)}{\sigma\left(x_{n-1}, x_{n}\right)}, \sigma\left(x_{n-1}, x_{n}\right)\right\} \\
& =\max \left\{\sigma\left(x_{n}, x_{n+1}\right), \sigma\left(x_{n-1}, x_{n}\right)\right\} .
\end{aligned}
$$

Suppose that for some $n \in \mathbb{N}$

$$
\max \left\{\sigma\left(x_{n}, x_{n+1}\right), \sigma\left(x_{n-1}, x_{n}\right)\right\}=\sigma\left(x_{n}, x_{n+1}\right) .
$$

Since $\sigma\left(x_{n}, x_{n+1}\right)>0$, using the property (2) of the simulation function, we obtain

$$
\zeta\left(\alpha\left(x_{n-1}, x_{n}\right) \sigma\left(x_{n}, x_{n+1}\right), \sigma\left(x_{n}, x_{n+1}\right)\right)<0,
$$

which is a contradiction. It follows that $r\left(x_{n-1}, x_{n}\right)=\sigma\left(x_{n-1}, x_{n}\right)$ for all $n \in \mathbb{N}$, implying that

$$
\zeta\left(\alpha\left(x_{n-1}, x_{n}\right) \sigma\left(x_{n}, x_{n+1}\right), \sigma\left(x_{n-1}, x_{n}\right)\right) \geq 0, \quad \text { for all } n \in \mathbb{N} .
$$


Using $\left(\zeta_{2}\right)$, the inequality (3.15) yields that

$$
\sigma\left(x_{n}, x_{n+1}\right) \leq \sigma\left(x_{n-1}, x_{n}\right), \quad \text { for all } n \in \mathbb{N} .
$$

Hence, $\left\{\sigma\left(x_{n}, x_{n+1}\right)\right\}$ is a non-increasing sequence, bounded from below, converging to some $L \geq 0$. Suppose that $L>0$. Taking the upper limit as $n \rightarrow \infty$ in (3.15), using $\left(\zeta_{3}\right)$, we get

$$
0 \leq \limsup _{n \rightarrow \infty} \zeta\left(\alpha\left(x_{n}, x_{n-1}\right) \sigma\left(x_{n}, x_{n+1}\right), \psi\left(\sigma\left(x_{n-1}, x_{n}\right)\right)\right)<0,
$$

which is a contradiction. Hence, we conclude that $\lim _{n \rightarrow \infty} \sigma\left(x_{n}, x_{n+1}\right)=0$.

In order to prove that $\left\{x_{n}\right\}$ is a Cauchy sequence, suppose the contrary. Then, as in the proof of Theorem 3.1, there exist $\epsilon>0$ and subsequences $\left\{x_{m_{k}}\right\}$ and $\left\{x_{m_{k}}\right\}$ of $\left\{x_{n}\right\}$, so that for $n_{k}>m_{k}>k$ we have

$$
\sigma\left(x_{m_{k}}, x_{n_{k}}\right) \geq \epsilon \text { and } \sigma\left(x_{m_{k}}, x_{n_{k}-1}\right)<\epsilon .
$$

Also, in the same way, the following inequalities hold:

$$
\begin{aligned}
\lim _{k \rightarrow \infty} \sigma\left(x_{m_{k}}, x_{n_{k}}\right) & =\lim _{k \rightarrow \infty} \sigma\left(x_{n_{k}-1}, x_{m_{k}-1}\right)=\epsilon, \\
\lim _{k \rightarrow \infty} \sigma\left(x_{m_{k}-1}, x_{n_{k}}\right) & =\lim _{k \rightarrow \infty} \sigma\left(x_{n_{k}-1}, x_{m_{k}}\right)=\epsilon .
\end{aligned}
$$

Since $T$ is triangular weakly- $\alpha$-admissible, we derive that

$$
\alpha\left(x_{n}, x_{m}\right) \geq 1, \quad \text { for all } n, m \in \mathbb{N}_{0} \text { with } n>m .
$$

Thus, we have

$$
\alpha\left(x_{m_{k}}, x_{n_{k}}\right) \geq 1 \text { and } \sigma\left(x_{m_{k}}, T x_{m_{k}-1}\right)=\sigma\left(x_{n_{k}}, T x_{n_{k}-1}\right)=\sigma(U, V),
$$

for all $k \in \mathbb{N}$. Since $T$ is a generalized $\alpha$ - $\zeta$-contraction, the obtained relations (3.17) imply

$$
0 \leq \zeta\left(\alpha\left(x_{m_{k}-1}, x_{n_{k}-1}\right) \sigma\left(x_{m_{k}}, x_{n_{k}}\right), r\left(x_{m_{k}-1}, x_{n_{k}-1}\right)\right), \quad \text { for all } k \in \mathbb{N} .
$$

Since

$$
r\left(x_{m_{k}-1}, x_{n_{k}-1}\right)=\max \left\{\frac{\sigma\left(x_{m_{k}-1}, x_{m_{k}}\right) \sigma\left(x_{n_{k}-1}, x_{n_{k}}\right)}{\sigma\left(x_{m_{k}-1}, x_{n_{k}-1}\right)}, \sigma\left(x_{m_{k}-1}, x_{n_{k}-1}\right)\right\},
$$

taking limits of both sides of (3.18), we conclude that $\lim _{k \rightarrow \infty} r\left(x_{m_{k}-1}, x_{n_{k}-1}\right)=\epsilon$. Letting $k \rightarrow \infty$ and keeping (3.16) and $\left(\zeta_{3}\right)$ in mind, we get

$$
0 \leq \limsup _{n \rightarrow \infty} \zeta\left(\alpha\left(x_{m_{k}-1}, x_{n_{k}-1}\right) \sigma\left(x_{m_{k}}, x_{n_{k}}\right), r\left(x_{m_{k}-1}, x_{n_{k}-1}\right)\right)<0
$$

which is a contradiction. Thus, we conclude that the sequence $\left\{x_{n}\right\}$ is Cauchy in $U$.

The final step of the proof is the same as for Theorem 3.1. 


\section{Corollaries and Examples}

Using Example 2.1, it is possible to get a number of consequences of our main results by choosing the simulation function $\zeta$ and $\alpha(x, y)$ in a proper way. We skip making such a list of corollaries since they seem clear. We just state the following one as a sample

Corollary 4.1. Let $(X, \sigma)$ be a metric-like space, $U$ and $V$ be two non-empty subsets of $X$ and $\alpha: X \times X \rightarrow \mathbb{R}_{0}^{+}, \psi \in \Psi$. Suppose that $T: U \rightarrow V$ is a given $\alpha$-proximal admissible mapping such that

$$
\alpha(x, y) \geq 1 \text { and } \sigma(u, T x)=\sigma(v, T y)=\sigma(U, V) \Rightarrow \alpha(x, y) \sigma(u, v) \leq \psi(\sigma(x, y))),
$$

for all $x, y, u, v \in U$. Suppose also

(a) $T$ is triangular weakly- $\alpha$-admissible;

(b) $U$ is closed with respect to the topology induced by $\tau_{\sigma}$;

(c) $T\left(U_{0}\right) \subset V_{0}$;

(d) there exist $x_{0}, x_{1} \in U$ such that $\sigma\left(x_{1}, T x_{0}\right)=\sigma(U, V)$ and $\alpha\left(x_{0}, x_{1}\right) \geq 1$;

(e) $T$ is continuous or $(\mathrm{P})$ holds.

Then, $T$ has a best proximity point.

In particular, if the given space $(X, \sigma)$ is also endowed with a partial order $\preceq$, by taking

$$
\alpha(x, y) \geq 1 \Leftrightarrow x \succeq y
$$

one can get standard variations of the given results in a partially ordered space.

The following illustrative examples show how our results can be used for certain mappings acting in metric-like spaces.

Example 4.1. Consider $X=\{a, b, c, d\}$ equipped with $\sigma: X \times X \rightarrow \mathbb{R}_{0}^{+}$defined by

$$
\begin{aligned}
& \sigma(a, a)=\frac{1}{2}, \quad \sigma(b, b)=0, \quad \sigma(c, c)=2, \quad \sigma(d, d)=\frac{1}{3}, \quad \sigma(a, b)=3, \\
& \sigma(a, c)=\frac{5}{2}, \quad \sigma(a, d)=\frac{3}{2} \quad \sigma(b, c)=2, \quad \sigma(b, d)=\frac{3}{2}, \quad \sigma(c, d)=\frac{5}{2},
\end{aligned}
$$

and $\sigma(x, y)=\sigma(y, x)$ for $x, y \in X$. It is clear that $(X, \sigma)$ is a complete metric-like space. Take $U=\{b, c\}$ and $V=\{c, d\}$. Consider the mapping $T: U \rightarrow V$ defined by $T b=d$, and $T c=c$. Remark that $\sigma(U, V)=\sigma(b, d)=\frac{3}{2}$. Also, $U_{0}=\{b\}$ and $V_{0}=\{d\}$. Note that $T\left(U_{0}\right) \subseteq V_{0}$. Take $\psi(t)=\frac{5}{6} t$, and $\zeta(t, s)=\frac{3}{4} s-t$ for all $t, s \geq 0$. Define $\alpha: X \times X \rightarrow \mathbb{R}_{0}^{+}$by

$$
\alpha(x, y)= \begin{cases}1, & x, y \in U \\ 0, & \text { otherwise }\end{cases}
$$

Let $x, y, u, v \in U$ be such that

$$
\alpha(x, y) \geq 1 \text { and } \sigma(u, T x)=\sigma(v, T y)=\sigma(U, V)=\frac{3}{2} .
$$


Then, necessarily, we have $x=y=u=v=b$. So, $\alpha(u, v) \geq 1$, that is, $T$ is $\alpha$-proximal admissible.

We need to prove that $T$ is an $\alpha-\psi-\zeta$ contraction. By the previous conclusion, the only case to be checked is when $x=y=u=v=b$. Then we have

$$
\zeta(\alpha(b, b) \sigma(b, b), \psi(\sigma(b, b)))=\zeta(1 \cdot 0, \psi(0))=0 .
$$

Thus, all the conditions of Theorem 3.1 are satisfied. So $T$ has a best proximity point (which is $z=b$ ). On the other hand, e.g., Corollary 2.2 (with $k=2$ ) of [4] is not applicable for the standard metric.

Example 4.2. Consider the set $X=\{a, b, c, d\}$ equipped with the following complete metric-like $\sigma$ :

$$
\begin{gathered}
\sigma(a, a)=\sigma(b, b)=\frac{1}{4}, \quad \sigma(c, c)=\sigma(d, d)=2 \\
\sigma(a, b)=\sigma(c, d)=\frac{1}{2}, \quad \sigma(a, c)=\sigma(b, d)=1, \quad \sigma(a, d)=\sigma(b, c)=\frac{3}{2},
\end{gathered}
$$

and $\sigma(x, y)=\sigma(y, x)$ for all $x, y \in X$. Let $U=\{a, b\}$ and $V=\{c, d\}$; then $\sigma(U, V)=$ 1, $U_{0}=U$ and $V_{0}=V$. Consider, further, the mappings $T: U \rightarrow V$ given by $T a=c$, $T b=c, \alpha: X \times X \rightarrow[0,+\infty)$ given by

$$
\alpha(x, y)= \begin{cases}1, & \text { if } x, y \in U \\ 0, & \text { otherwise }\end{cases}
$$

and $\zeta \in Z$ given by $\zeta(t, s)=s-\frac{2+t}{1+t} t$. Let us check that the mapping $T$ is a generalized $\alpha$ - $\zeta$-contraction. Let $x, y, u, v \in U$ be such that $x \neq y, \alpha(x, y) \geq 1$, $\sigma(u, T x)=\sigma(v, T y)=1$. Then it must be $u=v=a$ and either $x=a, y=b$ or $x=b$, $y=a$. In both cases, it is $\alpha(u, v) \geq 1$. In order to check condition (3.14), it is enough to consider the case $x=a, y=b, u=v=a$ (the other is treated symmetrically). Then,

$$
\begin{aligned}
\zeta(\alpha(x, y) \sigma(u, v), r(x, y)) & =\zeta\left(1 \cdot \frac{1}{4}, \max \left\{\frac{1}{2}, \frac{\frac{1}{4} \cdot \frac{1}{2}}{\frac{1}{2}}\right\}\right)=\zeta\left(\frac{1}{4}, \frac{1}{2}\right) \\
& =\frac{1}{2}-\frac{2+\frac{1}{4}}{1+\frac{1}{4}} \cdot \frac{1}{4}=\frac{1}{20}>0,
\end{aligned}
$$

and the condition is satisfied. All other conditions of Theorem 3.4 are fulfilled, hence, we conclude that the mapping $T$ has a best proximity point (which is $z=a$ ).

\section{Application to Best Proximity Results on a Metric-like Space with A GRAPH}

Throughout this section, $(X, \sigma)$ will denote a metric-like space and $G=(V(G), E(G))$ will be a directed graph such that its set of vertices $V(G)=X$ and the set of edges $E(G)$ contains all loops, i.e., $\Delta:=\{(x ; x): x \in X\} \subseteq E(G)$. We need in the sequel the following hypothesis: 
$\left(P_{G}\right)$ if a sequence $\left\{u_{n}\right\}$ in $X$ converges to $u \in A$ such that $\left(u_{n}, u_{n+1}\right) \in E(G)$, then there is a subsequence $\left\{u_{n(k)}\right\}$ of $\left\{u_{n}\right\}$ with $\left(u_{n(k)}, u\right) \in E(G)$ for all $k$.

Definition 5.1. Let $U$ and $V$ be two non-empty subsets of $X$ and $\alpha: X \times X \rightarrow \mathbb{R}_{0}^{+}$. We say that $T: U \rightarrow V$ is a $G$-proximal mapping if

$$
\left.\begin{array}{c}
(x, y) \in E(G), \quad \alpha(x, y) \geq 1, \\
\sigma(u, T x)=\sigma(v, T y)=\sigma(U, V)
\end{array}\right\} \Rightarrow(u, v) \in E(G)
$$

for all $x, y, u, v \in U$.

Definition 5.2 ([8,10]). Let $U$ and $V$ be two non-empty subsets of $X$, let $T: U \rightarrow V$ be a mapping and $\alpha: X \times X \rightarrow \mathbb{R}_{0}^{+}$be a function. We say that $T$ is triangular weakly- $G$-admissible if

$$
\alpha(x, y) \in E(G) \text { and } \alpha(y, z) \in E(G) \Rightarrow \alpha(x, z) \in E(G) .
$$

Corollary 5.1. Let $U$ and $V$ be two non-empty subsets of $X$ and $\psi \in \Psi$. Suppose that $T: U \rightarrow V$ is a mapping such that

$$
\sigma(T x, T y) \leq \psi(\sigma(x, y))
$$

for all $x, y \in U$ such that $(x, y) \in E(G)$. Suppose also:

(a) $T$ is triangular weakly-G-admissible;

(b) $T\left(U_{0}\right) \subset V_{0}$;

(c) there exist $x_{0}, x_{1} \in U$ such that $\sigma\left(x_{1}, T x_{0}\right)=\sigma(U, V)$ and $\left(x_{0}, x_{1}\right) \in E(G)$;

(d) $T$ is continuous or $\left(R_{G}\right)$ holds.

Then, $T$ has a best proximity point.

Proof. It suffices to consider $\alpha: X \times X \rightarrow \mathbb{R}_{0}^{+}$such that

$$
\alpha(x, y)= \begin{cases}1, & \text { if }(x, y) \in E(G) \\ 0, & \text { if not. }\end{cases}
$$

All the hypotheses of Corollary 4.1 are satisfied.

In this way, we can derive all results and consequences of the paper [15], extending them to partially ordered metric-like spaces. Similarly, we can extend the frame of several other existing results from, e.g., [3, 10,12,16].

Acknowledgements. The authors are thankful to the referee for his/her careful reading of the article.

\section{REFERENCES}

[1] H. H. Alsulami, E. Karapinar, F. Khojasteh and A. F. R.-L. de Hierro, A proposal to the study of contractions in quasi-metric spaces, Discrete Dyn. Nat. Soc. 2014 (2014), Article ID 269286.

[2] A. Amini-Harandi, Metric-like spaces, partial metric spaces and fixed points, Fixed Point Theory Appl. 2012(204) (2012), 10 pages. 
[3] N. Bilgili, E. Karapinar and B. Samet, Generalized $\alpha-\psi$-contractive mappings in quasi-metric spaces and related fixed-point theorems, J. Inequal. Appl. 2014(36) (2014), 15 pages.

[4] S. Chandok and M. Postolache, Fixed point theorem for weakly Chatterjea-type cyclic contractions, Fixed Point Theory Appl. 2013(28) (2013), 9 pages.

[5] A. F. R.-L. de Hierro, E. Karapinar, C. R.-L. de Hierro and J. Martínez-Moreno, Coincidence point theorems on metric spaces via simulation functions, J. Comput. Appl. Math. 75 (2015), 345-355.

[6] M. Jleli, E. Karapinar and B. Samet, Best proximity points for generalized $\alpha-\psi$-proximal contractive type mappings, J. Appl. Math. 2013 (2013), Article ID 534127, 10 pages.

[7] E. Karapinar, Fixed points results via simulation functions, Filomat 30(8) (2016), 2343-2350 .

[8] E. Karapinar, H. H. Alsulami and M. Noorwali, Some extensions for Geraghty type contractive mappings, J. Inequal. Appl. 2015(303) (2015), 22 pages.

[9] E. Karapinar and F. Khojasteh, An approach to best proximity points via simulation functions, J. Fixed Point Theory Appl. 19 (2017), 1983-1995.

[10] E. Karapinar, P. Kuman and P. Salimi, On $\alpha-\psi$-Meir-Keeler contractive mappings, Fixed Point Theory Appl. 2013 (2013), Article ID 94.

[11] E. Karapinar and P. Salimi, Dislocated metric space to metric spaces with some fixed point theorems, Fixed Point Theory Appl. 2013 (2013), Article ID 222.

[12] E. Karapinar and B. Samet, Fixed point theorems for generalized $\alpha-\psi$ contractive type mappings and applications, Abstr. Appl. Anal. 2012 (2012), 17 pages.

[13] F. Khojasteh, S. Shukla and S. Radenović, A new approach to the study of fixed point theorems via simulation functions, Filomat 29 (2015), 1189-1194.

[14] S. Radenović, Z. Kadelburg, D. Jandrlić and A. Jandrlić, Some results on weak contraction maps, Bull. Iranian Math. Soc. 38 (2012), 625-645.

[15] B. Samet, Best proximity point results in partially ordered metric spaces via simulation functions, Fixed Point Theory Appl. 2015(232) (2015), 15 pages.

[16] B. Samet, C. Vetro and P. Vetro, Fixed point theorem for $\alpha-\psi$ contractive type mappings, Nonlinear Anal. 75 (2012), 2154-2165.

${ }^{1}$ Department of Mathematics,

ICFAI UNIVERSITY,

RAipur, ChHATtisgarh-490042, INDIA

Email address: gvvjagan1@gmail.com

${ }^{2}$ Department of Mathematics,

SCHOOL OF Advanced SCIENCES,

Vellore Institute of TeChNology,

VELLORE-632014, TN, INDIA

Email address: drhknashine@gmail.com, hemant.nashine@vit.ac.in

${ }^{3}$ FACUlty of Mathematics,

UNIVERSITY OF BELGRADE,

Studentski trg 16, 11000 Beograd, Serbia

Email address: kadelbur@matf.bg.ac.rs

*Corresponding Author 